\title{
Incidentes na assistência das parturientes e recém-nascidos: perspectivas das enfermeiras e médicos
}

\author{
Incidents in the care provided to parturient women and newborns: the perspective of nurses and
} physicians

Incidentes en la assistencia de parturientas y recién nacidos: perspectivas de las enfermeras y médicos

\author{
Giullia Taldo Rodrigues ${ }^{1}$ (C) \\ Adriana Lenho de Figueiredo Pereira ${ }^{1}$ (C) \\ Priscila da Silva Almeida Pessanha ${ }^{1}$ (c) \\ Lucia Helena Garcia Penna ${ }^{1}$ (B)
}

1. Universidade do Estado do Rio de Janeiro (UERJ). Rio de Janeiro, RJ, Brasil.
Autor correspondente: Adriana Lenho de Pereira. E-mail: adrianalenho.uerj@gmail.com.

Recebido em 20/03/2020.

Aprovado em 20/05/2020.

\section{Resumo}

Objetivo: descrever os incidentes na assistência das parturientes e recém-nascidos, seus fatores contribuintes e medidas preventivas na perspectiva das enfermeiras e médicos. Método: pesquisa qualitativa em maternidade pública do Município do Rio de Janeiro, com Núcleo de Segurança do Paciente registrado. Realizaram-se entrevistas com 18 enfermeiras e médicos atuantes no centro obstétrico e duas enfermeiras integrantes desse Núcleo, de fevereiro a abril de 2019. Utilizaram-se referenciais da Análise de Conteúdo Temática e Segurança do Paciente. Resultados: os principais incidentes são erros na identificação dos pacientes, quedas da mãe e do bebê e eventos danosos causados pelas práticas inadequadas; desrespeito e maus-tratos às parturientes; ausência de notificações dos eventos; restrições de pessoal e recursos; limitações no conhecimento da equipe e apoio da gestão às ações de segurança do paciente. As medidas de prevenção são pertinentes às adequações e às melhorias da estrutura e processo de assistência e gerência dos cuidados. Conclusão e implicações para a prática: os incidentes são erros e danos, cuja prevenção requer qualificação do processo e estrutura da assistência, comprometimento dos profissionais e gerentes com a segurança das pacientes, e mudanças na cultura organizacional que também abarquem o enfrentamento da violência institucional obstétrica.

Palavras-chave: Dano ao Paciente; Parto; Segurança do Paciente; Cultura Organizacional; Qualidade da Assistência à Saúde.

\begin{abstract}
Objective: to describe the incidents in the care provided to parturient women and newborns, their contributing factors, and preventive measures, from the perspective of nurses and physicians. Method: qualitative study conducted in a public maternity hospital in the city of Rio de Janeiro, RJ, Brazil, with a registered Patient Safety Center. Interviews were held with 18 nurses and physicians working at the obstetric center and two nurses from the patient safety center, from February to April of 2019. The Thematic Content Analysis and Patient Safety references were used. Results: the main incidents were errors in patient identification, mother and newborn falls, and harmful events caused by inappropriate practices, disrespect and mistreatment of parturients, women, lack of incident reporting, insufficient personnel and resources, limited knowledge of the team, and lack of support on the part of management to patient safety actions.. Preventive measures are needed to adjust and improve the care structure, process, and management. Conclusion and implications for practice: the incidents are errors and harm, the prevention of which requires qualification of the care process and structure, the commitment of professionals and managers to patient safety, and changes in the organizational culture that also encompass coping with institutional obstetric violence.
\end{abstract}

Keywords: Patient Harm; Parturition; Patient Safety; Organizational Culture; Quality of Health Care.

\section{Resumen}

Objetivo: describir incidentes en el cuidado de parturientas y recién nacidos, los factores que contribuyen a ellos y las medidas preventivas para evitarlos, desde la perspectiva de enfermeras y médicos. Método: investigación cualitativa en una maternidad pública del municipio de Río de Janeiro que cuenta con un Núcleo de Seguridad del Paciente registrado. Se realizaron entrevistas a 18 enfermeras y médicos que trabajan en el centro obstétrico y dos enfermeras que forman parte del NSP, entre febrero y abril de 2019. Se utilizaron como referencia los aportes del Análisis de Contenido Temático y de Seguridad del Paciente. Resultados: los principales incidentes corresponden a errores en la identificación de los pacientes, caídas de la madre y el bebé y eventos dañinos causados por prácticas inadecuadas; falta de respeto y maltrato a las parturientas; ausencia de notificaciones de los eventos; restricciones de personal y recursos; limitaciones en los conocimientos del equipo y en el apoyo a la gestión de las acciones de seguridad del paciente. Las medidas de prevención son relevantes para los ajustes y mejoras en la estructura, el proceso y la gestión de los cuidados. Conclusión e implicaciones para la práctica: los incidentes son errores y daños, cuya prevención requiere la evaluación del proceso y la estructura de los cuidados, el compromiso con la seguridad de los pacientes por parte de los profesionales y gestores, además de los cambios en la cultura organizacional, que incluyen el enfrentamiento a la violencia obstétrica institucional.

Palabras clave: Daño al paciente; Parto; Seguridad del Paciente; Cultura Organizacional; Calidad de la Atención de Salud. 


\section{INTRODUÇÃO}

A segurança do paciente é uma dimensão essencial da qualidade em saúde para a Organização Mundial da Saúde (OMS) e visa reduzir danos associados à assistência e melhorar os indicadores de saúde. A qualidade é alcançada quando se obtém os melhores resultados e experiências possíveis, enquanto a segurança busca evitar ao máximo os erros e danos nos cuidados à saúde. Portanto, a baixa qualidade corresponde as falhas na obtenção de resultados exitosos e a baixa segurança na ocorrência de danos evitáveis aos pacientes. ${ }^{1-3}$

A avaliação da qualidade em saúde contempla a análise da estrutura, processo e resultado da assistência. A estrutura abarca os recursos e materiais necessários para prover o cuidado; o processo envolve a prática assistencial dos profissionais e o resultado é reflexo do nível de adequação da estrutura e processo assistenciais. A avaliação contínua da qualidade permite a correção dos erros antes que eles ocorram e provoquem incidentes aos pacientes e, assim, agir de forma preventiva. ${ }^{3,4}$

Cabe esclarecer que incidente é um evento ou circunstância evitável que causa ou não dano ao paciente. $O$ incidente sem dano envolve as circunstâncias notificáveis, que são qualquer tipo de evento, situação ou processo com potencial danoso significativo, a exemplo das falhas na comunicação, inadequação de pessoal e deficiências na manutenção de equipamentos. Ao ser detectado com antecedência e não alcançar o paciente, denomina-se quase-erro ou near-miss. Por fim, os incidentes danosos ou eventos adversos (EA) causam necessariamente algum tipo de dano ao paciente, físico, psicológico ou social. ${ }^{4,5}$ Estima-se que a prevalência dos EA seja de 1 a $4 \%$ em unidades da atenção obstétrica. . $^{3,4}$

A busca pela qualidade dos cuidados no parto e adequação do atendimento é essencial para reduzir a mortalidade materna. $A$ promoção da materna segura pautada na prevenção quaternária, ou seja, sem iatrogenias e danos é um enfoque promissor na redução das morbidades e mortes, bem como para aumentar a satisfação das mulheres com assistência recebida. ${ }^{6}$

O Brasil tem investido políticas e programas nesta direção, como a criação do Programa Nacional de Segurança do Paciente (PNSP) em 2013, para possibilitar avanços na melhoria da qualidade e segurança da assistência à saúde prestada a sua população. Para tal, determinou-se a implantação do Núcleo de Segurança do Paciente (NSP) em todos os serviços de saúde para promover e apoiar as ações institucionais de segurança do paciente..$^{3,5}$

Em face da criação do PNSP, a Agência Nacional de Vigilância Sanitária (ANVISA) do Ministério da Saúde divulgou documento de referência para os serviços da atenção materna e neonatal em 2014, visando contemplar as especificidades dessa área de atenção, avançar na prevenção de resultados adversos provenientes do processo assistencial e melhorar os indicadores de saúde deste segmento populacional. ${ }^{6}$

Apesar dessas ações governamentais, ainda se verificam práticas inadequadas que impactam negativamente na qualidade e segurança da assistência e nos resultados de saúde materna e neonatal. As taxas de cesarianas e intervenções desnecessárias em partos vaginais continuam elevadas e impactam negativamente na mortalidade materna no país. ${ }^{7,8}$

Além desses desafios, verificou-se que, entre dez mil puérperas, $2 \%$ delas receberam assistência sem danos e a prevalência de danos evitáveis foi elevada, como episiotomia $(87,7 \%)$, cesarianas $(55,4 \%)$ e internações de recém-nascidos a termo em Unidade Neonatal Intensiva (UTI-N) (7,7\%), o que revela ser premente investir em melhorias na segurança e qualidade da assistência materna. ${ }^{6,8}$

Diante desses problemas associados com a segurança e qualidade da assistência ao parto e das ações programáticas para redução dos danos às mulheres e aos seus filhos, o presente estudo partiu da seguinte questão norteadora: Quais os incidentes na assistência das parturientes e recém-nascidos, seus fatores contribuintes e medidas preventivas segundo as enfermeiras e médicos?

O estudo objetivou descrever os incidentes na assistência das parturientes e recém-nascidos, seus fatores contribuintes e medidas preventivas na perspectiva das enfermeiras e médicos.

\section{MÉTODO}

Estudo de abordagem qualitativa, tipo descritivo e realizado em maternidade pública de grande porte no Município do Rio de Janeiro, que é referência para gestantes de risco habitual e com morbidade associada, e com atendimento anual de mais de 5.000 nascimentos, segundo os dados de nascimento sistematizados pela instituição.

A opção por essa maternidade foi devido ao fato dela constar na relação nominal dos serviços de saúde que dispõem de Núcleo de Segurança do Paciente (NSP) e divulgada na página eletrônica da ANVISA, bem como por dispor de enfermeiras obstétricas atuantes na assistência ao parto normal. O NSP dessa maternidade foi criado no segundo semestre de 2016, mas as suas atividades não estavam ocorrendo regularmente no período da coleta de dados da pesquisa, de fevereiro a abril de 2019.

Os participantes do estudo foram enfermeiras e médicos dos setores Centro Obstétrico (CO) e NSP da instituição. Adotaramse os seguintes critérios de inclusão: ser enfermeira ou médico atuante nos cuidados às parturientes no $\mathrm{CO}$ e/ou integrante da equipe do NSP no serviço. Excluíram-se os profissionais com seis meses ou menos de trabalho nesses setores da maternidade.

Para recrutamento das enfermeiras e médicos, inicialmente, duas integrantes da equipe de pesquisa expuseram os objetivos da pesquisa às chefias da instituição e às equipes do $\mathrm{CO}$ antes da coleta de dados. Posteriormente, por ocasião da coleta de dados, essa exposição oral foi repetida sempre que necessário, a fim de aumentar a adesão desses profissionais ao estudo.

Esses profissionais foram selecionados de forma intencional e conforme a observação do envolvimento ou liderança dos mesmos na assistência prestada no CO e/ou ações do NSP, buscandose também observar a representatividade proporcional entre as equipes do $\mathrm{CO}$, captar primeiramente os profissionais com mais 
tempo de trabalho na instituição e verificar a disponibilidade dos mesmos para conceder a entrevista no ambiente laboral.

Após explicação dos objetivos da pesquisa a cada profissiona selecionado, verificaram-se os critérios de inclusão e exclusão acima descritos. Alguns participantes solicitaram agendamento prévio, sendo que nenhum deles se recusou a conceder entrevista. Os depoimentos foram individuais e durante os momentos de pausas ou intervalos das atividades laborais, sendo concedidos em local privativo na instituição ou sala de reuniões da equipe.

Utilizou-se roteiro de entrevista semiestruturado com questões destinadas à caracterização do perfil profissional dos participantes e seis perguntas abertas acerca das ações desenvolvidas pelo NSP; motivos da ocorrência de incidentes na assistência ao parto; tipos de danos à mulher e ao bebê por ocasião do parto; notificações dos incidentes pela equipe; medidas de prevenção e ações necessárias à assistência segura da mulher e bebê.

As entrevistas foram conduzidas pela primeira autora, integrante da equipe de pesquisa e previamente treinada e supervisionada por uma pesquisadora doutora mais experiente, que revisou os depoimentos gravados e suas transcrições. Posteriormente, procedeu-se a análise propriamente dita e conforme o referencial da Análise de Conteúdo Temática (ACT) de Lawrence Bardin. ${ }^{9}$

$\mathrm{Na}$ pré-análise da ACT, cada depoimento foi transcrito, organizado e lido de forma flutuante para identificação dos recortes do texto pertinentes aos propósitos do estudo. Posteriormente, houve a exploração do material por meio do agrupamento das unidades de significação e conforme a equivalência semântica dos códigos descobertos, culminando na construção das categorias temáticas. Quando novos códigos ou temas não emergiram dessa fase da análise, as entrevistas cessaram. Dois membros da equipe de pesquisa não participantes da coleta de dados revisaram essas etapas. Por fim, o tratamento dos resultados ocorreu por meio de inferências e interpretações, ${ }^{9}$ sendo essas norteadas pelos conceitos e pressupostos da Segurança do Paciente aplicados na assistência ao parto e nascimento.

O estudo atendeu os princípios éticos e foi aprovado pela Comissão de Ética em Pesquisa da Universidade do Estado do Rio de Janeiro, sob o Parecer no 2.533.376 e CAAE n 81896117.0 .0000 .5282 . Todos os participantes do estudo assinaram o Termo de Consentimento Livre e Esclarecido, sendo aplicada codificação segundo a categoria profissional, setor de atuação e ordem de concessão das Entrevistas (E), a exemplo de Enfermeira do CO, E1; Médico do CO, E2; Enfermeira do NSP, E3; assim por diante.

\section{RESULTADOS}

Dos 20 participantes do estudo, 12 enfermeiras atuantes no CO compuseram um grupo constituído por seis enfermeiras que exercem os cuidados gerais de enfermagem e seis enfermeiras obstétricas responsáveis pela assistência ao trabalho de parto e parto normal. Essas profissionais têm de 24 a 51 anos de idade e de um a 25 anos de trabalho na maternidade, cuja média de tempo é de 6,9 anos, sendo a maioria do sexo feminino. Dos seis médicos atuantes no $\mathrm{CO}$, a maioria é do sexo masculino, quatro são obstetras e dois pediatras. Eles têm de 27 a 68 anos de idade e a média de tempo de trabalho na instituição de 10,4 anos, que variou de 1,5 a 27 anos.

As duas enfermeiras integrantes do NSP desde a sua criação são mulheres com idade de 41 e 42 anos, e especialistas na área de Saúde Pública, sendo que uma era especialista também em Segurança do Paciente. Essas profissionais trabalhavam na maternidade há 7 e 16 anos.

A ACT originou duas categorias que estão descritas a seguir, sendo elas: incidentes de segurança e fatores contribuintes à sua ocorrência em parturientes e recém-nascidos, e medidas de prevenção dos incidentes na assistência do parto e nascimento.

\section{Incidentes de segurança e fatores contribuintes à sua ocorrência em parturientes e recém-nascidos}

As enfermeiras e médicos caracterizam os incidentes como erros ou falhas ocorridas durante a assistência das mulheres e seus filhos por ocasião do parto e nascimento, sendo relacionados com as situações de queda; falhas na identificação dos pacientes; troca de medicamentos e da prescrição, e ausências ou incompletudes nos registros dos cuidados, como mencionam as falas a seguir.

[...] Acho que o principal [incidente] é a queda do bebêe da mãe, o mais comum mesmo. (Enfermeira do CO, E12)

[...] Vivenciar aqui eu não me recordo, mas já ouvi falar de troca de medicação e de uma paciente que caiu da maca [...]. (Médico do CO E10)

[...] Incidente é questão da identificação do paciente que, às vezes, não foi identificado direito [...]. Já chegou prescrição trocada de uma paciente com a outra [...]. (Enfermeira do CO, E14)

[...] Lembrei outra situação recente de falha de registro e vacinação equivocada, do bebê receber a vacina num dia e outra [dose] no dia seguinte [...] (Enfermeira do NSP, E16)

Os incidentes com danos ou EA nas mulheres e recémnascidos foram caracterizados como danos físicos, principalmente, e decorrentes da inadequação do conhecimento, atitudes e práticas profissionais, como manobras e intervenções obstétricas desnecessárias ou mal indicadas que causam lesões traumáticas e, por vezes, com desfechos graves para a mãe ou do bebê.

[...]Eu acho que a negligência é o principal fator, mas, às vezes, até mesmo a falta de informação dos profissionais. (Médico do CO, E.9)

[...]Excesso de intervenções com essa mulher [...]. Dano ao bebê, eu acredito que seja em relação à administração de ocitocina e às manobras feitas durante o parto. (Enfermeira do CO, E3)

[...]Eu acho que é a falta de qualidade da assistência mesmo. Às vezes, a mulher fica um pouco largada quando 
a maternidade está cheia [...], aí, alguns danos e incidentes podem ocorrer. (Enfermeira do CO, E5)

Além desses danos, as falas destacam as situações de violência institucional obstétrica sofridas pelas mulheres na assistência ao parto e que provocam danos emocionais e físicos, como expressam a enfermeira e o médico.

[...] É a queda e a violência obstétrica[...]. É violência obstétrica quando os profissionais aplicam [manobra de] Kristeller, por exemplo, e há fratura de clavícula do bebê e lesão do plexo [braquial]. (Enfermeira do CO, E13)

[...] acontece é o que se chama de violência obstétrica. A gente percebe alguns profissionais que desconhecem o parto humanizado e falam coisas que consideramos como violência à parturiente (Enfermeira do CO, E18)

[...] Então, você pode ter todo o tipo de dano: dano ao períneo e dano ao emocional da paciente, e dano também ao recém-nato a depender das práticas não adequadas na assistência ao parto. (Médico do CO, E.6)

Alguns participantes caracterizam os problemas sociais e clínicos da mulher como incidentes, sendo relacionados com riscos associados e complicações maternas, como morbidades prévias à gestação; inadequação no acesso à assistência prénatal e falta de rede social de apoio à parturiente.

[...] Os danos são riscos associados ao parto [...] lacerações, "pico" de pressão, descompensação da paciente [...], parada de progressão, falha da motricidade uterina [...] Há paciente que não tem acompanhante [...], quando tem, a família é pouco estruturada [...], e aquela que nem vai no pré-natal [...]. (Médico do $\mathrm{CO}, \mathrm{E} 4)$

[...] Na verdade, os motivos são vários das complicações da paciente, como pré-eclâmpsia, diabetes e comorbidades associadas à gestação. [...] O que mais complica é não ter CTI materno [na maternidade] para a compensar essas pacientes. (Médico do CO, E2)

Outras deficiências na estrutura e processo do serviço corroboram para a ocorrência dos incidentes na visão dos profissionais, como a interrupção das atividades do NSP; ausência de protocolos, vigilância e notificação dos incidentes; deficiências na capacitação profissional, recursos humanos e equipamentos.

[...] Eu acho que falta um trabalho especifico que oriente melhor as pessoas para que esses erros não aconteçam [...], protocolo e treinamento da equipe são importantes. (Médico do CO, E10)

[...] nossas camas tipo PPP [Pré-parto, Parto e Pós-parto] estão quase todas quebradas, as cadeiras de rodas são antigas e [...] isso aumenta o risco de acidentes e queda da paciente. (Enfermeira do CO, E12)

[...] Percebi dificuldades em desenvolver ações [do NSP] porque não conseguimos garantir as pessoas realmente voltadas para isso. (Enfermeira do NSP, E16)

[...] No momento, não temos aqui ações especificas do NSP [...], mas teve uma ação efetiva na implementação do protocolo de identificação correta do paciente principalmente. (Enfermeira do NSP E.17)

Além dessas limitações organizacionais, as falas das enfermeiras e médicos expressam conhecimento limitado sobre a segurança do paciente e desconhecimento acerca do NSP na instituição e dos incidentes como eventos notificáveis.

[...] A principal ação que conheço é identificação do paciente[...], mas também não deixar na cama e ter um bercinho para acomodá-lo. (Enfermeira do CO, E.5)

[...] Então... eu nunca fui apresentada a um Núcleo de Segurança aqui. (Enfermeira do CO, E.20)

[...] Não sei, mas também se tiver [notificação de incidentes] eu desconheço, porque nunca aconteceu comigo e, aí, também nem procuro saber. (Médico do CO, E.7)

\section{Melhorias no processo e estrutura para prevenção dos incidentes na assistência do parto e nascimento}

$\mathrm{Na}$ ótica das enfermeiras e médicos, as medidas preventivas dos incidentes envolvem a melhoria na assistência das parturientes e seus filhos, como a adequação assistência por meio do uso das boas práticas e qualificação dos cuidados e comunicação da equipe.

[...] Acho que as medidas de prevenção focam muito na qualidade da assistência [...]. Então, é observar a hora de ouro e fazer a ausculta corretamente, conforme preconiza a OMS. (Enfermeira do CO, E.5)

[...] As boas práticas são interligadas com a segurança [...]. Então, as medidas de prevenção são o acolhimento; identificação da mulher; registro adequado [...]; fluxo certo em relação a linha de cuidado e comunicação entre os profissionais para se ter sucesso no cuidado. (Enfermeira do CO, E.20)

Eu acho que a orientação é o principal, orientar bem as mães e a equipe [...]. Não só a prevenção dos incidentes, mas a promoção da saúde durante o pré-natal [...], falar como é um trabalho de parto [...] cuidado com a mãe, neném e depois com o pediatra [...] e tudo mais. (Médico do CO, E.4) 
Além dessas medidas, os participantes também consideram que a prevenção dos incidentes requer um conjunto de melhorias no processo e estrutura da assistência, tais como: capacitação dos profissionais; protocolo de comunicação; processo de trabalho qualificado; cuidado centrado na mulher; vigilância dos incidentes; gestão envolvida com a segurança do paciente; funcionamento do NSP; recursos apropriados; manutenção equipamentos e quantidade de pessoal suficiente à demanda de cuidados.

[...] Dentro da instituição, ter mais recursos, medicamentos, exames e profissionais, [...] entender o protagonismo da mulher e a presença do acompanhante [...], precisamos trabalhar muito a comunicação efetiva, o protocolo de comunicação é urgente, mas precisa de um Núcleo operante, né. (Enfermeira do CO, E.15)

[...] as medidas de prevenção são de natureza pessoal, os profissionais se manterem atualizados, com hábito permanente de estudar e buscar a literatura e os congressos. (Médico do CO, E.6)

[...] Acho que trabalhar com indicadores é importante porque apontam aquilo que o gestor precisa se debruçar para melhorar o processo de trabalho [...] e reduzir esses danos. (Enfermeira do NSP, E.16)

[...]treinamento em equipe e reflexão sobre a prática [...] focar no cuidado centrado no paciente [...], mas precisa de uma agenda prioritária de gestão que englobe todas as chefias deste serviço. (Enfermeira do NSP, E.17)

\section{DISCUSSÃO}

Os erros e eventos adversos são um dos maiores desafios nos sistemas de saúde em âmbito global. Estima-se que um em cada dez pacientes sofram EA durante $o$ atendimento em hospitais, sendo a maioria evitável. Esses danos sofridos prolongam o tempo de internação e podem causar a morte dos pacientes. ${ }^{10}$

Em quarenta e uma maternidades da Espanha, estimouse incidência acumulada de EA em 3,6\%, cujas causas foram intervenções ou procedimentos cirúrgicos $(59,4 \%)$ e administração de medicamentos (12,5\%). Esses danos prolongaram a internação hospitalar e causaram reinternações em 13,3\% das mulheres, apesar de não terem implicado em morbidade grave ou morte martena. ${ }^{11}$

Em maternidade pública brasileira, a maioria das notificações de incidentes foi procedente da clínica obstétrica (45,8\%), préparto $(19,3 \%)$ e a UTIN $(15,1 \%)$ no período de 2017 a 2019 . O incidente mais notificado foi o erro na identificação das mulheres (27\%) e neonatos (18,4\%), seguido da omissão de cuidados ou dose de medicamento (16,9\%); quedas dos recém-nascidos $(15,3 \%)$; queda das mulheres $(10,8 \%)$ e falta de materiais hospitalares (8,4\%). ${ }^{12}$ Esses tipos de incidentes também foram destacados nas falas das enfermeiras e médicos entrevistados no presente estudo.
A identificação correta do paciente é prioritária na prevenção de incidentes e melhoria da segurança do paciente. Em outra maternidade pública do País, verificou-se que $10 \%$ das mulheres internadas foram expostas ao risco potencial de erros assistenciais em virtude do primeiro nome e do último sobrenome serem idênticos. Em hospital brasileiro, estimou-se taxa de identificação incorreta dos bebês em 7,8\% em unidade neonatal. Portanto, as competências institucionais e profissionais devem ser desenvolvidas para melhoria da conformidade da pulseira de identificação e efetividade dessa medida, inclusive com a participação ativa das mães e acompanhantes. ${ }^{13}$

As quedas dos pacientes são os incidentes mais relatados em hospitais britânicos, cuja taxa média é de 6,63 quedas por 1.000 dias de leito ocupado e frequência de 30 a $50 \%$ de lesões físicas e de 1 a $3 \%$ de fraturas, sobretudo em pessoas idosas. ${ }^{14}$ Em recém-nascidos, estima-se de 1,6 a 4,4 quedas por 10.000 nascimentos em hospitais americanos, tendo a exaustão materna como principal fator contribuinte, seguido dos equipamentos hospitalares como camas e berços hospitalares. Esses eventos também são considerados frequentes pelas enfermeiras e médicos entrevistados. Além dos riscos à saúde do recémnascido, desde traumatismos menores até a morte, as quedas causam também sofrimento emocional e insatisfação nas mães, assim como sentimento de culpa e medo por advertências ou litígio entre os profissionais. ${ }^{15}$

Além dessas repercussões negativas, os eventos adversos graves no parto e nascimento podem ter efeito catastrófico e duradouro sobre a vida da mãe ou filho. Estima-se que 5\% das grávidas vivenciaram problemas na qualidade da assistência no trabalho de parto e parto em hospitais americanos, sendo a maioria deles associados aos erros do profissional (87\%) e, portanto, classificados como incidentes evitavéis. ${ }^{16}$

Apesar das lesões ou traumas graves no sistema orgânico dos pacientes serem associadas aos EA, as enfermeiras e médicos consideram que o desrespeito e os maus-tratos são comportamentos associados à violência institucional e que causam danos emocionais e físicos no parto. A experiência negativa nesse momento pode ter efeitos debilitantes profundos na autoestima e saúde mental das mulheres; contribui para o risco de depressão pós-parto e transtorno de estresse pós-traumático, impactando no apego e vínculo maternos, além de repercutir nas decisões reprodutivas subsequentes, como a desistência de ter outro filho; adiamento de uma futura gravidez; medo do parto normal e preferência de cesariana na próxima gestação. ${ }^{1,17}$

Portanto, debates e estudos acerca da segurança precisam contemplar os comportamentos, atitudes e práticas desrespeitosas e potencialmente iatrogênicas para as parturientes e recémnascidos, pois as enfermeiras e médicos sugerem haver uma possível correlação e efeito sinérgico entre o fenômeno da violência institucional e os problemas de segurança na assistência.

A violência institucional no parto é um problema crítico na atenção obstétrica brasileira e em outros países. Estudo com 2.138 mulheres americanas constatou que uma a cada seis mulheres têm experiências de desrespeito e maus-tratos, como 
a perda da autonomia; atendimento com gritos, repreensões ou ameaças proferidas pelos profissionais e percepção de ser ignorada e não respondida aos pedidos de ajuda. Os relatos de maus-tratos foram mais frequentes nos hospitais do que quando o parto ocorreu no domicílio da mulher e em centro de parto normal, assim como nas parturientes indígenas, latinas e pretas em comparação com as mulheres de pele branca. ${ }^{18}$

Portanto, as grávidas estão expostas a riscos e danos decorrentes da assistência inadequada, desrespeitosa, discriminatória e iatrogênica, o que coloca como pauta prioritária a promoção do cuidado materno respeitoso, pautado nos direitos humanos e centrado na dignidade da pessoa, bem como a necessidade de incluir indicadores sensíveis e confiáveis de aferição dos abusos e maus-tratos no parto, a fim de viabilizar mudanças nos contextos, práticas e culturas inseguras no cuidado à saúde $e$ relacionadas com a violência ao gênero feminino. ${ }^{6,8}$

Em virtude desse cenário desafiador, políticas têm sido instituídas para alcançar uma cultura de segurança positiva em saúde. ${ }^{5,8}$ No entanto, a cultura organizacional é vista como um fator crítico no avanço das melhorias pretendidas em face da tendência de se observar posturas de aceitação do padrão cultural estabelecido na assistência e trabalho em saúde. A cultura se expressa "no como se faz" e "no que se opta tolerar" nas organizações e, desse modo, correlaciona-se com o nível de alcance dos resultados esperados. ${ }^{19}$

A segurança tem relação com a cultura organizacional ao promover o engajamento da equipe, favorecer mudanças relevantes e reconhecer a assistência como um sistema adaptativo complexo e resultante dos diferentes processos e atores implicados. Assim sendo, a cultura de segurança positiva valoriza os acertos ou resultados exitosos obtidos, aprendizagem com os erros, participação na resolução das falhas nos processos de cuidados e colaboração ativa nas ações de melhoria. ${ }^{16,19}$

Nessa perspectiva, a segurança e qualidade contemplam componentes essenciais, como as diretrizes clínicas baseadas em evidências e listas de verificação padronizadas; processos eficazes de comunicação e transferência da responsabilidade pelos cuidados ao paciente para a sua continuidade; trabalho em equipe comprometido, cooperativo e respeitoso entre os seus membros; educação permanente e capacitação profissional contínua, inclusive por meio de simulações, além do compromentimento de gerentes e gestores com as ações de melhoria da segurança e qualidade na instituição. A maior parte desses componentes foram citados pelas enfermeiras e médicos como medidas necessárias à prevenção dos incidentes. 3,16,20

Além de externarem a compreensão desses componentes essenciais, as enfermeiras e médicos expressam limitações e restrições no processo e estrutura assistencial da maternidade que prejudicam a prevenção dos incidentes, como a descontinuidade das atividades do NPS; inexistência de notificação e monitoramento dos incidentes; ausência de protocolos de segurança; restrições nos recursos humanos e materiais, e limitações no conhecimento profissional, sendo estabelecidas também associações equivocadas entre os incidentes e as morbidades maternas.
Portanto, a partir dos pontos de vistas dessas enfermeiras e médicos, infere-se que o NSP não foi efetivamente implementado na maternidade. Apesar desse fator restritivo, parece que as atividades inicialmente desenvolvidas pelo NSP foram capazes de sensibilizar ou mobilizar a equipe para a primeira meta da segurança, a identificação correta das mulheres e bebês, bem como podem ter despertado a percepção dos mesmos quanto às necessidades de avanços na cultura organizacional e competências individuais e coletivas para a segurança da assistência.

O fato do NSP não estar operante na instituição, até o término deste estudo, pode ter dificultado a construção do sistema de notificação de incidentes, sem o qual as iniciativas de prevenção de incidentes sofrem prejuízos. Por outro lado, as inadequações dos recursos e equipamentos apontadas pelos entrevistados podem ser consequentes ao subfinanciamento crônico do sistema público de saúde no País, ${ }^{6}$ o que denota ser fator macroestrutural limitante para a efetividade das metas programáticas acerca da segurança e qualidade em saúde materna e neonatal, como aquelas preconizadas pelo PNSP.

Outro ponto crítico na estrutura é a visão desses profissionais acerca das atividades do NSP não integrarem a pauta de prioridades da gestão local. Essa limitação aponta para a necessidade da ampliação das estratégias de capacitação e sensibilização para gerentes e gestores das maternidades, uma vez que a mudança pretendida depende do engajamento de todos os atores envolvidos, inclusive de representantes dos usuários dos serviços de saúde, movimentos sociais e de mulheres. ${ }^{9,21}$ Essas estratégias podem favorecer a compreensão das dinâmicas afetivas, cognitivas, atitudinais e culturais dos indivíduos e das lógicas presentes no contexto de implementação das ações de segurança, como as estruturas, processos, comportamentos, práticas e valores político-organizacionais desses espaços sociais de cuidados à saúde. ${ }^{16,22}$

Por fim, destaca-se que os resultados aqui descritos devem ser apreciados de forma parcimoniosa devido às limitações do estudo, uma vez que abarcou as perspectivas de um grupo particular de enfermeiras e médicos que não representam a diversidade de realidades, contextos e pontos de vistas acerca da segurança da assistência ao parto e nascimento em âmbito local e nacional. No entanto, espera-se que os resultados aqui descritos contribuam para o debate acerca dos desafios na prevenção de incidentes e dos avanços necessários à assistência e à pesquisa sobre a temática, inclusive com a utilização de métodos mais abrangentes e participativos que contemplem outros contextos, cenários e perspectivas da equipe multiprofissional em saúde materna e neonatal.

\section{CONCLUSÕES E IMPLICAÇÕES PARA A PRÁTICA}

Os principais incidentes relatados pelas enfermeiras e médicos são os erros na identificação, quedas e danos à mãe e ao bebê. Os danos físicos e emocionais são ocasionados pelas inadequações no processo e estrutura da assistência às parturientes e aos recém-nascidos, bem como relacionados 
com os seguintes fatores contribuintes: 1) uso de práticas inadequadas e atitudes de violência pelos profissionais; 2) inoperância do NSP e ausência de protocolos de segurança, notificação e monitoramento de incidentes na instituição; 3) restrições de pessoal, recursos e manutenção de equipamentos; 4) limitações no conhecimento da equipe e apoio da gestão às ações de segurança e NSP no serviço.

Em face dessas inadequações, foram propostas medidas preventivas que envolvem adequações e melhorias no processo e estrutura assistenciais, inclusive mudanças na cultura organizacional em virtude da correlação estabelecida entre os incidentes de segurança e o fenômeno da violência institucional obstétrica às mulheres em trabalho de parto e parto.

Essa correlação pode denotar complexidade adicional na abordagem da segurança das pacientes em hospitais da atenção obstétrica, que é o atravessamento das iniquidades de gênero feminino relacionadas com posturas de desrespeito e maus-tratos às mulheres grávidas nesses espaços assistenciais. Sugere-se que os processos destinados à mudança da cultura organizacional em maternidades contemplem os problemas da insegurança do atendimento juntamente com os desafios da violência institucional, a fim de ampliar a efetividade das políticas de saúde e iniciativas locais destinadas à melhoria da qualidade e segurança da assistência no parto e nascimento.

\section{CONTRIBUIÇÕES DOS AUTORES}

Desenho do estudo. Adriana Lenho de Figueiredo Pereira. Coleta de dados. Adriana Lenho de Figueiredo Pereira. Giullia Taldo Rodrigues.

Análise de dados. Adriana Lenho de Figueiredo Pereira. Giullia Taldo Rodrigues. Adriana Lenho de Figueiredo Pereira.

Interpretação dos resultados. Adriana Lenho de Figueiredo Pereira. Priscila da Silva Almeida Pessanha. Lucia Helena Garcia Penna.

Redação e revisão crítica do manuscrito. Aprovação da versão final do artigo. Responsabilidade por todos os aspectos do conteúdo e a integridade do artigo publicado. Adriana Lenho de Figueiredo Pereira. Giullia Taldo Rodrigues. Priscila da Silva Almeida Pessanha. Lucia Helena Garcia Penna.

\section{EDITOR ASSOCIADO}

\section{Ana Luiza de Oliveira Carvalho}

\section{REFERÊNCIAS}

1. Lyberg A, Dahl B, Haruna M, Takegata M, Severinsson E. Links between patient safety and fear of childbirth: a meta-study of qualitative research. Nurs Open. 2019;6(1):18-29. http://dx.doi.org/10.1002/nop2.186. PMid:30534391.

2. Pettker CM. Systematic approaches to adverse events in obstetrics, part I: event identification and classification. Semin Perinatol. 2017;41(3):1515. http://dx.doi.org/10.1053/j.semperi.2017.03.003. PMid:28549789.

3. Minuzzi AP, Salum NC, Locks MOH, Amante LN, Matos E. Contributions of healthcare staff to promote patient safety in intensive care. Esc Anna Nery. 2016;20(1):121-9. http://dx.doi.org/10.5935/1414-8145.20160017.
4. Sagawa MR, Silva AEB C, Lima JC, Bezerra ALQ, Costa NN, Sousa MRG et al. Analysis of notifiable circumstances: incidents that may compromise patient safety. Cogitare Enferm. 2019;24:e61984. http:// dx.doi.org/10.5380/ce.v24i0.61984.

5. Agência Nacional de Vigilância Sanitária (BR). Serviços de atenção materna e neonatal: segurança e qualidade. Brasília: ANVISA; 2014.

6. Salgado HO, Souza JP, Sandall J, Diniz CSG. Patient safety in maternity care in brazil: the maternity safety thermometer as a tool to improve the quality of care. Rev Bras Ginecol Obstet. 2017;39(5):199-201. http:// dx.doi.org/10.1055/s-0037-1602704. PMid:28476064

7. Silva BG, Lima NP, Silva SG, Antúnez SF, Seerig LM, Restrepo-Méndez MC et al. Maternal mortality in Brazil from 2001 to 2012: time trends and regional differences. Rev Bras Epidemiol.2016;19(3):484-93. http:// dx.doi.org/10.1590/1980-5497201600030002. PMid:27849265.

8. Salgado HO, Queiroz MR, Santos HG, Andreucci CB, Diniz CSG Using the maternity safety thermometer to estimate harm-free care in Southeast Brazil: a hospital-based cohort. Birth. 2019;46(4):583-91. http://dx.doi.org/10.1111/birt.12454. PMid:31579979.

9. Bardin L. Análise de conteúdo. São Paulo: Edições 70; 2011.

10. Asgari Dastjerdi H, Khorasani E, Yarmohammadian $\mathrm{MH}$, Ahmadzade MS. Evaluating the application of failure mode and effects analysis technique in hospital wards: a systematic review. J Inj Violence Res. 2017;9(1):51-60. http://dx.doi.org/10.5249/jivr.v9i1.794. PMid:28039688.

11. Aibar L, Rabanaque MJ, Aibar C, Aranaz JM, Mozas J. Patient safety and adverse events related with obstetric care. Arch Gynecol Obstet. 2015;291(4):825-30. http://dx.doi.org/10.1007/s00404-014-3474-3. PMid:25245667.

12. Silva WC, Santos SHD, Soares AN, Silva RA, Silva CO, Melo KC et al Análise da ocorrência de incidentes notificados no ambiente hospitalar de uma maternidade pública. Revista Eletrônica Acervo Saúde. 2019;(34):e1445. http://dx.doi.org/10.25248/reas.e1445.2019.

13. Hideco TT, Quadrado ERS, Tronchin DMR. Avaliação do risco de erro na identificação de mulheres numa maternidade pública. Rev Bras Enferm. 2018;71(1):120-5. http://dx.doi.org/10.1590/0034-7167-20170134. PMid:29324953.

14. Morris R, O'Riordan S. Prevention of falls in hospital. Clin Med 2017;17(4):360-2. http://dx.doi.org/10.7861/clinmedicine.17-4-360. PMid:28765417.

15. Lipke B, Gilbert G, Shimer H, Consenstein L, Aris C, Ponto L et al. Newborn safety bundle to prevent falls and promote safe sleep. MCN Am J Matern Child Nurs. 2018;43(1):32-7. http://dx.doi.org/10.1097/ NMC.0000000000000402. PMid:29045245.

16. Sabol B, Caughey AB. Quality improvement and patient safety on labor and delivery. Obstet Gynecol Clin North Am. 2017;44(4):667-78. http:// dx.doi.org/10.1016/j.ogc.2017.08.002. PMid:29078948.

17. Shorey S, Yang YY, Ang E. The impact of negative childbirth experience on future reproductive decisions: a quantitative systematic review. J Adv Nurs. 2018;74(6):1236-44. http://dx.doi.org/10.1111/jan.13534 PMid:29394456.

18. Vedam S, Stoll K, Taiwo TK, Rubashkin N, Cheyney M, Strauss N et al The Giving Voice to Mothers study: inequity and mistreatment during pregnancy and childbirth in the United States. Reprod Health. 2019;16(1):77. http://dx.doi.org/10.1186/s12978-019-0729-2. PMid:31182118.

19. Ruiter PJA. Implementing patient safety initiatives. Obstet Gynecol Clin North Am. 2019;46(2):281-92. http://dx.doi.org/10.1016/j.ogc.2019.01.005. PMid:31056130.

20. Alves M, Melo CL. Transferência de cuidado na perspectiva de profissionais de Enfermagem de um pronto-socorro. REME-Rev Min Enferm. 2019;23:e-1194. http://dx.doi.org/10.5935/1415-2762.20190042.

21. Alves MFT, Carvalho DS, Albuquerque GSC. Motivos para a não notificação de incidentes de segurança do paciente por profissionais de saúde: revisão integrativa. Cien Saude Colet. 2019;24(8):2895-908 http://dx.doi.org/10.1590/1413-81232018248.23912017.PMid:31389537.

22. Liberati EG, Tarrant C, Willars J, Draycott T, Winter C, Chew S et al. How to be a very safe maternity unit: an ethnographic study. Soc Sci Med. 2019;223:64-72. http://dx.doi.org/10.1016/j.socscimed.2019.01.035. PMid:30710763. 